\title{
Systemic Treatments for Metastatic Renal Cell Carcinoma: 10-Year Experience of Immunotherapy and Targeted Therapy
}

\author{
Sung Han Kim, MD, PhD \\ Weon Seo Park, MD, PhD ${ }^{1,2}$ \\ Sun Ho Kim, MD, PhD ${ }^{3}$ \\ Jae Young Joung, MD, $P h D^{1}$ \\ Ho Kyung Seo, MD, PhD' \\ Kang Hyun Lee, MD, PhD' \\ Jinsoo Chung, MD, PhD'
}

\section{Purpose}

The purpose of this study is to compare the outcomes of first-line systemic targeted therapy (TT) and immunotherapy (IT) in patients with metastatic renal cell carcinoma (mRCC).

\section{Materials and Methods}

This study was a retrospective review of the data of 262 patients treated with systemic IT or $\Pi$ with tyrosine kinase inhibitors between 2003 and 2013. The objective response rate (ORR), progression-free survival (PFS), and overall survival (OS) were assessed using Response Evaluation Criteria in Solid Tumor ver. 1.0 criteria and the Kaplan-Meier method with log-rank test.

Results

During the median 4.3-month treatment and the 24-month follow-up period, the ORR/PFS/OS of the overall first-line and second-line therapy were 41.9\%/8.1 months/16.8 months and 27.5\%/6.5 months/15.3 months, respectively. The first-line TT/IT/sequential IT had a PFS of 9.3/6.4/5.7 months and an OS of 15.8/16.5/40.6 months (all $p<0.05$ ). The second-line of TT/IT had a PFS of 7.1/2.1 months (both $p<0.05$ ) and an OS of 16.6/8.6 months $(p=0.636)$, respectively. Pazopanib provided the best median PFS of 11.0 months $(p<0.001)$ and a quadruple IT regimen had a superior PFS ( $p=0.522)$. For OS, sequential treatment with IT and TT was superior compared to treatment with either IT or TT alone (40.6/16.5/15.8 months, $p=0.014)$. The prognosis according to the Memorial Sloan Kettering Cancer Center model showed that favorable/intermediate/poor risk groups had a PFS of 8.5/10.4/2.3 months, and an OS of 43.1/20.4/5.6 months, respectively. The prognosis calculated using the Heng model showed that the favorable/intermediate/poor risk groups had a PFS of 9.2/3.9/2.7 months, and an OS of 32.4/16.5/6.1months, respectively (all $p<0.001$ ).

\section{Conclusion}

In patients with mRCC, TT provided a better PFS and OS compared with IT.
Center for Prostate Cancer,

Research Institute and

Hospital of National Cancer Center,

323 Ilsan-ro, Ilsandong-gu, Goyang 10408, Korea

Tel: 82-31-920-2456

Fax: $82-31-920-2474$

E-mail: cjs5225@ncc.re.kr

Received August 19, 2015

Accepted January 11, 2016

Published Online January 28, 2016
Key words

Immunotherapy, Molecular targeted therapy, Prognosis,

Renal cell carcinoma, Neoplasm metastasis

\section{Introduction}

Worldwide, metastases are observed in 20\%-30\% of patients with an initial diagnosis of renal cell carcinoma (RCC), even in those with localized RCC who undergo curative nephrectomy during follow-up. In the immunotherapy
(IT) era, cytokine-mediated IT using interferon alpha (IFN$\alpha$ ) or interleukin-2 (IL-2) or both were the first-line systemic treatment modalities for metastatic RCC (mRCC) [1,2]. However, the outcomes of IT were often unsatisfactory. Further attempts to augment cytokine-based IT activity (e.g., inclusion of vinblastine and 5-fluorouracil [5-FU] chemotherapy) led to slightly improved objective response rate (ORR) [3,4]. 
However, the efficacy of such combinations was likely due to the cytokine effect, because the addition of vinblastine did not improve the progression-free survival (PFS) or overall survival (OS) [3]. The reported median survival time of mRCC in the IT era was 12 months and the 2-year survival rate was $10 \%-20 \%$, mainly due to a low ORR of $10 \%-20 \%$, with long-term durable responses only observed in 5\%-7\% of patients undergoing high-dose IL-2-based therapy $[5,6]$.

Recent advances in understanding of the biology and genetics of RCC have led to the introduction of several novel multi-pathway targeted agents. Clinical trials in treatmentnaïve mRCC patients conducted in Western and Asian countries have shown that targeted therapy (TT) had superior efficacy when compared with cytokine-based IT [2,7]. In terms of PFS and OS, the clinical outcome of patients with $\mathrm{mRCC}$ has improved dramatically since the introduction of TT, which has now replaced IT as the standard systemic treatment for mRCC [1].

To facilitate prompt management strategies and achieve the optimal therapeutic response, physicians should be aware of the efficacies and limitations of systemic mono, combinational, and sequential therapies of diverse therapeutic agents for the treatment of mRCC. However, few large studies comparing the outcome of IT and TT in patients with mRCC have been reported, although small comparative studies have been conducted [8]. The current study assessed the outcome of systemic TT and IT administered to a large number of patients with mRCC over a 10-year period at a single Korean cancer institution.

\section{Materials and Methods}

\section{Patients}

Data from 262 patients with mRCC treated with systemic agents between 2003 and 2013 were evaluated retrospectively. Patients who were administered IT as a systemic therapy between 2003 and 2013 were selected from the prospectively recorded RCC database of the National Cancer Center Hospital, and those administered TT who were treated between 2006 and 2013 were selected. The clinicopathological data of all 262 patients are summarized in Table 1.

Of the 262 patients, 135 (51.5\%) underwent first-line IT regimens based on mono or combinational therapy with IFN- $\alpha$, IL-2, 5-FU, or vinblastine. The remaining 127 patients (48.5\%) underwent first-line systemic TT. Eligible patients were 18-75 years old. Patients with no follow-up history and who had incomplete RCC treatment histories were excluded.
Additional information about the clinical outcomes of 96 patients treated with either TT or IT as a sequential secondline systemic therapy was also evaluated.

\section{Treatment regimens}

The choice of IT or TT was at the discretion of the treating urologist (J.C.) and was based on the patient's histopathology and coverage by the National Health Insurance System. Combination IT comprised different combinations of subcutaneous recombinant human IL-2 (Aldesleukin, Proleukin, Chiron Corporation, Emeryville, CA) or recombinant human IFN- $\alpha$ (IFN-alpha-2a, Roferon-A, Hoffmann-La Roche, Nutley, NJ), and intravenous 5-FU (JW Pharm, Seoul, Korea) or vinblastine (United Pharm, Seoul, Korea). The triple or quadruple regimens were administered as follows: IL-2, 20 $\mathrm{MIU} / \mathrm{m}^{2}$ on days $3-5$ of weeks 1 and 4 , and $5 \mathrm{MIU} / \mathrm{m}^{2} \mathrm{IL}-2$ on days 1,3 , and 5 of weeks 2 and $3 ;$ IFN- $\alpha, 6 \mathrm{MIU} / \mathrm{m}^{2}$ on day 1 of weeks 1 and 4 and on days 1,3 , and 5 of weeks 2 and 3 , and $9 \mathrm{MIU} / \mathrm{m}^{2}$ on days 1,3 , and 5 of weeks $5-8 ; 5-\mathrm{FU}$, $750 \mathrm{mg} / \mathrm{m}^{2}$ once weekly during weeks 5-8; with (quadruple) or without vinblastine (triple) at $0.1 \mathrm{mg} / \mathrm{kg}$ once weekly during weeks 5-8. The dual combination regimen of vinblastine plus IFN- $\alpha$ was administered as IFN- $\alpha$ at $9 \mathrm{MIU} / \mathrm{m}^{2}$ on days 1,3 , and 5 each week, and vinblastine at $0.1 \mathrm{mg} / \mathrm{kg}$ every 3 weeks.

\section{Targeted therapy}

All TT was administered orally. For sunitinib, each cycle consisted of $50 \mathrm{mg} /$ day for 4 weeks followed by a 2-week hiatus. For sorafenib, each cycle consisted of consecutive 400 $\mathrm{mg}$ twice daily per week. For pazopanib, each cycle consisted of consecutive $800 \mathrm{mg}$ once daily per week. Subjects were administered consecutive $10 \mathrm{mg}$ of everolimus once a day per week and $5 \mathrm{mg}$ of axitinib twice a day per week as the second-line TT. The treatment response was assessed every 4-6 weeks for sorafenib, pazopanib, everolimus, and axitinib.

\section{Follow-up}

During follow-up, meticulous history taking, physical examination, routine blood tests, abdominal computed tomography $(\mathrm{CT})$, radionuclide bone scan, simple chest radiography, and/or chest $\mathrm{CT}$, and electrocardiography were performed at regular intervals. In addition, mandatory thyroid function tests and echocardiography were performed in patients scheduled to receive TT. All responses were evaluated on CT imaging according to the Response Evaluation Criteria in Solid Tumor (RECIST) ver. 1.0. Therapeutic responses were assessed at the end of each cycle (at 8 weeks for the triple and quadruple IT regimens) or every 3-4 cycles 
Table 1. Patient baseline demographics

Parameter

No. $(\%)(\mathrm{n}=262)$

Age, mean \pm SD (yr)

$57.3 \pm 11.6$

Sex (male/female)

$204(77.9) /$

$58(22.1)$

BMI, mean \pm SD $\left(\mathrm{kg} / \mathrm{m}^{2}\right)$

$23.4 \pm 3.2$

Underlying disease

Diabetes

$46(17.6)$

Hypertension

97 (37)

$2(0.8)$

$4(1.5)$

Cerebrovascular disease

254 (96.9)

8 (3.1)

$50-70 \%$

$23(11.2)$

131 (63.6)

$52(25.2)$

56

Unknown

$41(17)$

Favorable

Intermediate

$160(66.4)$

Poor

40 (16.6)

Unknown

22

Treatment duration, median (range, mo) $\quad 4.3$ (0.1-68.4)

Metastatic site

$\begin{array}{lc}\text { Lung } & 204(77.3) \\ \text { Liver } & 44(16.7) \\ \text { LN } & 116(43.9) \\ \text { Bone } & 89(33.7) \\ \text { Brain } & 23(8.7) \\ \text { Other } & 36(13.6)\end{array}$

Clinical T stage

T1 25 (9.5)

T2 $32(12.2)$

T3 $71(27.1)$

$\mathrm{T} 4 \quad 21(8)$

$\mathrm{Tx}$

115 (43.6)

Clinical N stage

N1 $37(14.1)$

$\mathrm{Nx} \quad 109(42)$

Renal embolization 16 (6.1)

Nephrectomy 124 (47.3)

Pathologic T stage

T1 $22(8.3)$

T2 $27(10.3)$

T3 $53(20.1)$

$\mathrm{T} 4 \quad 8$ (3)

Tx 152 (57.9)

Pathologic N stage

$\begin{array}{cc}\mathrm{N} 1 & 12(4.6) \\ \mathrm{Nx} & 154(59.1)\end{array}$

Table 1. Continued

Parameter

No. $(\%)(n=262)$

Fuhrmann grade

1 7 (2.7)

$242(16)$

$3 \quad 82(31.3)$

$4 \quad 35$ (13.4)

Unknown 96 (36.6)

Histology

Clear cell, pure $178(67.4)$

Mixed 34 (12.9)

Papillary $5(1.9)$

Chromophobe $2(0.8)$

Unclassified $\quad 5(1.9)$

Unknown 38 (15.1)

Primary treatment drug

Target therapy $127(48.5)$

Sunitinib $92(35.1)$

Sorafenib $17(6.5)$

Pazopanib $18(6.9)$

Immunotherapy 135 (51.5)

Secondary treatment drug $\quad 96$

Target therapy $83(86.5)$

Sunitinib $33(39.8)$

Sorafenib $23(27.7)$

Pazopanib 3 (3.6)

Everolimus $21(25.3)$

Axitinib 3 (3.6)

Immunotherapy 13 (13.5)

Second-line best response (RECIST criteria 1.0) 69 (100)

PD 28 (40.6)

SD $22(31.9)$

PR $16(23.2)$

CR $3(4.3)$

F/U loss or death 27

Second-line progression free survival, $\quad 6.5$ (4.9-8.0)

median (range, mo)

Target therapy/Immunotherapy, $\quad 7.1(5.8-8.4) /$

median (range, mo)

$2.1(1.8-2.4)$

Second-line overall survival, $\quad 15.3(8.9-21.7)$

median (range, mo)

Target therapy/Immunotherapy, $\quad 16.6$ (10-22.5)/

median (range, mo)

$8.6(0.1-27.5)$

SD, standard deviation; BMI, body mass index; MSKCC, Memorial Sloane Kettering Cancer Center; LN, lymph node; RECIST, Response Evaluation Criteria in Solid Tumors; PD, progressive disease; SD, stable disease; PR, partial response; $\mathrm{CR}$, complete response; F/U, follow-up. 
(at 9-12 weeks for the dual IT regimen). For TT, response was assessed at the end of two cycles and treatment was continued until disease progression.

\section{Statistical analyses}

Time to progression and death were assessed using Kaplan-Meier analysis with the log-rank test. The ORR, PFS, and OS of the first- and second-line therapies were assessed according to the systemic agent and the clinical outcomes of sequential treatment with IT and TT, and their prognostic stratifications, were also evaluated according to the Memorial Sloan Kettering Cancer Center (MSKCC) and Heng criteria $[9,10]$. Statistical analyses were performed using Stata software (Release 9.2, StataCorp., College Station, TX). A p-value of $<0.5$ was considered statistically significant.

\section{Ethical statements}

Following approval by the Institutional Review Board (IRB) of the Research Institute and Hospital National Cancer Center (IRB No. NCC2015-0212), patient record/information was anonymized and de-identified prior to analysis. All study protocols were conducted according to the ethical guidelines of the World Medical Association Declaration of Helsinki-Ethical Principles for Medical Research Involving Human Subjects. The requirement for written consent was waived by the IRB.

\section{Results}

During the treatment period (median duration, 4.3 months) and the 24-month follow-up period, the first-line ORR, PFS, and OS were $41.9 \%, 8.1$ months, and 16.8 months, respectively (Table 1). During these same periods, the second-line ORR, PFS, and OS were 27.5\%, 6.5 months, and 15.3 months, respectively. Significantly better prognostic outcomes of second-line PFS were observed in the TT group (7.1 months) compared with the IT group ( 2.1 months) ( $p=0.002)$. However, second-line OS was not significantly different between the TT (16.6 months) and IT (8.6 months) groups $(\mathrm{p}=0.636)$.

In subgroup analyses of first-line systemic therapies, the TT, IT, and sequential groups had a PFS of 9.3, 6.4, and 5.7 months ( $\mathrm{p}=0.819)$, respectively, and an OS of $15.8,16.5$, and 40.6 months $(p=0.014)$, respectively (Table 2 , Fig. 1$)$. Pazopanib provided the best median PFS response, followed by sorafenib, sunitinib, and IT ( $p=0.053)$ (Table 2, Fig. 2). The quadruple IT regimen had superior PFS to that of IT mono, dual, or triple regimens ( $\mathrm{p}=0.522)$ (Table 2). Among the 14 IT-treated patients who achieved a complete response (CR), eight $(5.9 \%)$ remained alive with a mean response duration of $104.5 \pm 42.8$ months (median, 123 months; range, 33.1 to 156.7 months); four of these $(3.15 \%)$ also received TT treatment. For the seven TT-treated patients who achieved a CR, four remained alive, with a median response duration of 22.8 months (range, 13.9 to 54.8 months) (Table 2).

For the risk-stratified subgroup analyses that were dependent on the MSKCC and Heng criteria (Table 3), the clinical outcomes of PFS for each stratified prognostic group were followed subsequently in order according to their consecutive risk groups. When stratified according to the MSKCC or Heng criteria, treatments had differential outcomes for median OS. For the favorable MSKCC risk group in the first-line therapy, TT showed a superior OS compared with IT or sequential IT plus TT ( $<<0.001)$ (Table 3). In contrast, for the favorable Heng risk group, IT had superior OS compared with TT or IT plus TT $(\mathrm{p}<0.001)$. Other prognostic outcomes of first-line therapies and PFS and OS of secondline therapies, as calculated using the MSKCC and Heng risk group models, are summarized in Table 3.

\section{Discussion}

Since the introduction of TT, the therapeutic regimen paradigm has shifted from IT to TT in mRCC. The prognoses have also improved dramatically in the clinical setting. In the current study, differences in prognoses in mRCC patients treated either with IT, TT, or sequential therapy as the firstand second-line systemic therapies were examined at a single center during 10 years.

The current study showed that the median PFS and OS of IT regimens was superior or equivocal to those observed in other Western studies on IT [2,5,6,11], and their ORR of $41.9 \%$ and disease control rate of $69.5 \%$ (31.1\% and $54.8 \%$; including 59 follow-up loss and death patients) were superior to those reported in a previous Korean study on IT $(18 \%$ and $51 \%$, respectively) (Table 1) [8]. The use of 5-FU or vinblastine chemotherapy in conjunction with IT increased the PFS by $0.5-3$ months in $40.0 \%$ of the patients undergoing IT treatment, and quadruple IT resulted in the best PFS (8.8 months) compared with all other IT combinations (5.8-6.2 months) (Table 2). This suggests that the clinical benefit of an IT with the addition of a chemotherapy regimen should be carefully considered in terms of whether the increased survival gains would outweigh the likely increase in adverse events.

Patients previously treated with high-dose IL-2 achieved 
Table 2. Comparison of clinicopathological parameters between first-line IT and TT

\begin{tabular}{|c|c|c|c|}
\hline Parameter & IT $(n=135)$ & TT (n=127) & p-value \\
\hline Age, mean $\pm S D(y r)$ & $56.1 \pm 11.9$ & $58.5 \pm 11.2$ & 0.101 \\
\hline Sex (male/female) & $104 / 31(77 / 23)$ & 100/ 27 (78.7/21.3) & 0.768 \\
\hline $\mathrm{BMI}$, mean $\pm \mathrm{SD}\left(\mathrm{kg} / \mathrm{m}^{2}\right)$ & $23.6 \pm 3.5$ & $23.3 \pm 2.7$ & 0.421 \\
\hline \multicolumn{4}{|l|}{ Underlying disease } \\
\hline Diabetes & $18(13.3)$ & $28(22.0)$ & 0.075 \\
\hline Hypertension & $42(31.3)$ & $55(43.3)$ & 0.055 \\
\hline Ischemic heart disease & 0 & $2(1.6)$ & 0.234 \\
\hline Cerebrovascular disease & 0 & $4(3.1)$ & 0.054 \\
\hline \multicolumn{4}{|l|}{ Karnofsky performance score } \\
\hline$>80 \%$ & $131(97.0)$ & $123(96.9)$ & 0.481 \\
\hline $50-70 \%$ & $4(3.0)$ & $4(3.1)$ & \\
\hline \multicolumn{4}{|l|}{ MSKCC risk group } \\
\hline Favorable & $10(7.4)$ & $13(10.3)$ & 0.814 \\
\hline Intermediate & $63(46.7)$ & $68(53.5)$ & \\
\hline Poor & $27(20.0)$ & $25(19.7)$ & \\
\hline Unknown & $35(25.9)$ & $21(16.5)$ & \\
\hline \multicolumn{4}{|l|}{ Heng risk group } \\
\hline Favorable & $20(14.8)$ & $21(16.5)$ & 0.795 \\
\hline Intermediate & $87(64.4)$ & $73(57.5)$ & \\
\hline Poor & $21(15.6)$ & $19(15.0)$ & \\
\hline Unknown & $7(5.2)$ & $14(11.0)$ & \\
\hline Treatment duration, mean \pm SD (mo) & $7.2 \pm 9.8$ & $9.0 \pm 12.3$ & 0.204 \\
\hline \multicolumn{4}{|l|}{ Metastatic site } \\
\hline Lung & $102(75.6)$ & $102(80.3)$ & 0.633 \\
\hline Liver & $20(14.8)$ & $24(18.9)$ & 0.496 \\
\hline LN & $52(38.5)$ & $64(50.4)$ & 0.099 \\
\hline Bone & $45(33.3)$ & $44(34.6)$ & 0.675 \\
\hline Brain & $9(6.7)$ & $14(11.0)$ & 0.433 \\
\hline Other & 15 (11.1) & $21(16.5)$ & 0.421 \\
\hline \multicolumn{4}{|l|}{ Clinical T stage } \\
\hline $\mathrm{T} 1$ & $13(9.6)$ & $12(9.4)$ & 0.049 \\
\hline T2 & $18(13.3)$ & $14(11.0)$ & \\
\hline $\mathrm{T} 3$ & $36(26.7)$ & $35(27.6)$ & \\
\hline $\mathrm{T} 4$ & $10(7.4)$ & $11(8.7)$ & \\
\hline Tx & $60(44.4)$ & $55(43.3)$ & \\
\hline \multicolumn{4}{|l|}{ Clinical N stage } \\
\hline N1 & $18(13.3)$ & $19(15.0)$ & 0.017 \\
\hline $\mathrm{Nx}$ & $58(43.0)$ & $51(40.2)$ & \\
\hline Renal embolization & $11(8.1)$ & $5(3.9)$ & 0.199 \\
\hline Nephrectomy & $101(74.8)$ & $67(52.8)$ & $<0.001$ \\
\hline Primary renal tumor in situ & $25(18.5)$ & $56(43.3)$ & $<0.001$ \\
\hline \multicolumn{4}{|l|}{ Fuhrmann grade } \\
\hline 1 & $2(1.5)$ & $5(3.9)$ & 0.116 \\
\hline 2 & $16(11.9)$ & $26(20.5)$ & \\
\hline 3 & $47(34.8)$ & $35(27.6)$ & \\
\hline 4 & 15 (11.1) & $20(15.7)$ & \\
\hline Unknown & $65(48.1)$ & $41(32.3)$ & \\
\hline
\end{tabular}


Table 2. Continued

\begin{tabular}{|c|c|c|c|}
\hline Parameter & IT & TT & p-value \\
\hline \multicolumn{4}{|l|}{ Histology } \\
\hline Clear cell, pure & $84(62.2)$ & $94(74.0)$ & 0.066 \\
\hline Mixed & $6(4.4)$ & $13(10.2)$ & \\
\hline Papillary & $9(6.7)$ & $6(4.7)$ & \\
\hline Chromophobe & 0 & $1(0.7)$ & \\
\hline Unclassified & 0 & $5(3.9)$ & \\
\hline Unknown & $36(26.7)$ & $10(7.9)$ & \\
\hline \multirow{4}{*}{ Primary treating drug } & IL-2: $11(8.1)$ & Sunitinib: 92 (35.1) & NA \\
\hline & IFN- $\alpha: 65$ (48.1) & Sorafenib: 17 (6.5) & \\
\hline & IL-2+IFN- $\alpha: 57$ (42.2) & Pazopanib: 18 (6.9) & \\
\hline & Others: 2 (1.5) & & \\
\hline \multicolumn{4}{|l|}{ Best overall response } \\
\hline CR & $14(10.4)$ & $7(5.6)$ & 0.242 \\
\hline PR & $28(20.7)$ & $36(28.3)$ & \\
\hline SD & $32(23.7)$ & $24(18.9)$ & \\
\hline PD & $34(25.2)$ & $28(22.0)$ & \\
\hline F/U loss or death & $29(21.5)$ & $32(25.2)$ & \\
\hline \multirow[t]{5}{*}{ PFS, median (range, mo) } & $6.4(4.2-8.6)$ & $9.3(6.8-11.7)$ & 0.026 \\
\hline & $5.7(1.9-9.6)^{a)}$ & & 0.819 \\
\hline & IL-2: 3.3 (0.1-24.3) & Sunitinib: 8.5 (0.2-68.4) & 0.053 \\
\hline & IFN- $\alpha: 5.8$ (0.1-65.6) & Sorafenib: 9.0 (0.1-22.3) & \\
\hline & IL-2+IFN- $\alpha: 16.3$ (0.4-20.2) & Pazopanib: 11.0 (0.5-36.4) & \\
\hline \multicolumn{4}{|l|}{ PFS of IT, median (range) } \\
\hline Monotherapy $(\mathrm{n}=48)$ & $5.8(0.1-65.6)$ & NA & 0.522 \\
\hline Dual therapy $(n=33)$ & $7.5(0.1-22.5)$ & & \\
\hline Triple therapy $(\mathrm{n}=46)$ & $6.2(0.7-15.5)$ & & \\
\hline Quadruple therapy $(\mathrm{n}=8)$ & $8.8(1.7-20.2)$ & & \\
\hline \multirow[t]{2}{*}{ OS, median (range, mo) } & $16.5(0.7-156.7)$ & $15.8(0.3-78.4)$ & 0.452 \\
\hline & $\left.40.6(38.2-43.0)^{\mathrm{a}}\right)$ & & 0.014 \\
\hline
\end{tabular}

IT, immunotherapy; TT, targeted therapy; SD, standard deviation; BMI, body mass index; MSKCC, Memorial Sloane Kettering Cancer Center; LN, lymph node; Tx, treatment; IL-2, interleukin 2; NA, not available; IFN- $\alpha$, interferon $\alpha$; CR, complete response; $\mathrm{PR}$, partial response; $\mathrm{SD}$, stable disease; $\mathrm{PD}$, progressive disease; $\mathrm{F} / \mathrm{U}$, follow-up; PFS, progression-free survival; OS, overall survival. a)Progression-free survival and overall survival for IT alone/IT with subsequent TT versus TT alone.

a $15 \%$ of ORR with a median duration of 54 months [6], suggesting that high-dose IL-2 treatment may have been curative in a small minority of patients [5]. There are no clear guidelines regarding patient selection; however, retrospective studies have suggested that IL-2 was more efficacious in patients with a clear cell histology and favorable prognosis [12]. IFN- $\alpha$, another systemic immunotherapeutic agent for mRCC, has consistently demonstrated low but reproducible responses (10\%-20\%) with occasional durable responses with a PFS and OS of 5-5.6 and 4.1-21.8 months, respectively $[4,13,14]$. This is similar to the data of the patients in the current study undergoing IFN- $\alpha$ monotherapy who had similar PFS and OS (5.8 months) (Table 2) and 19.3 months (19.3 months, data not shown).
A combination of IL-2 and IFN- $\alpha$ showed a better clinical outcome in terms of ORR and PFS when compared with IL2 monotherapy (Table 2). In addition, sequential therapy with the combined IT and TT regimen resulted in a better OS (40.6 months) compared to single TT (15.8 months) and single IT (16.5 months) treatment regimens $(\mathrm{p}=0.014)$ (Table 2$)$. However, this outstanding sequential therapy OS may have resulted from our selection of patients who were capable of surviving IT prior to TT and were expected to have a good performance based on favorable prognostic modeling with a fairly low tumor burden and a history of nephrectomy. In particular, patients with rapid progression might be excluded from participation before beginning TT. Therefore, conditional survival may be an important methodology 

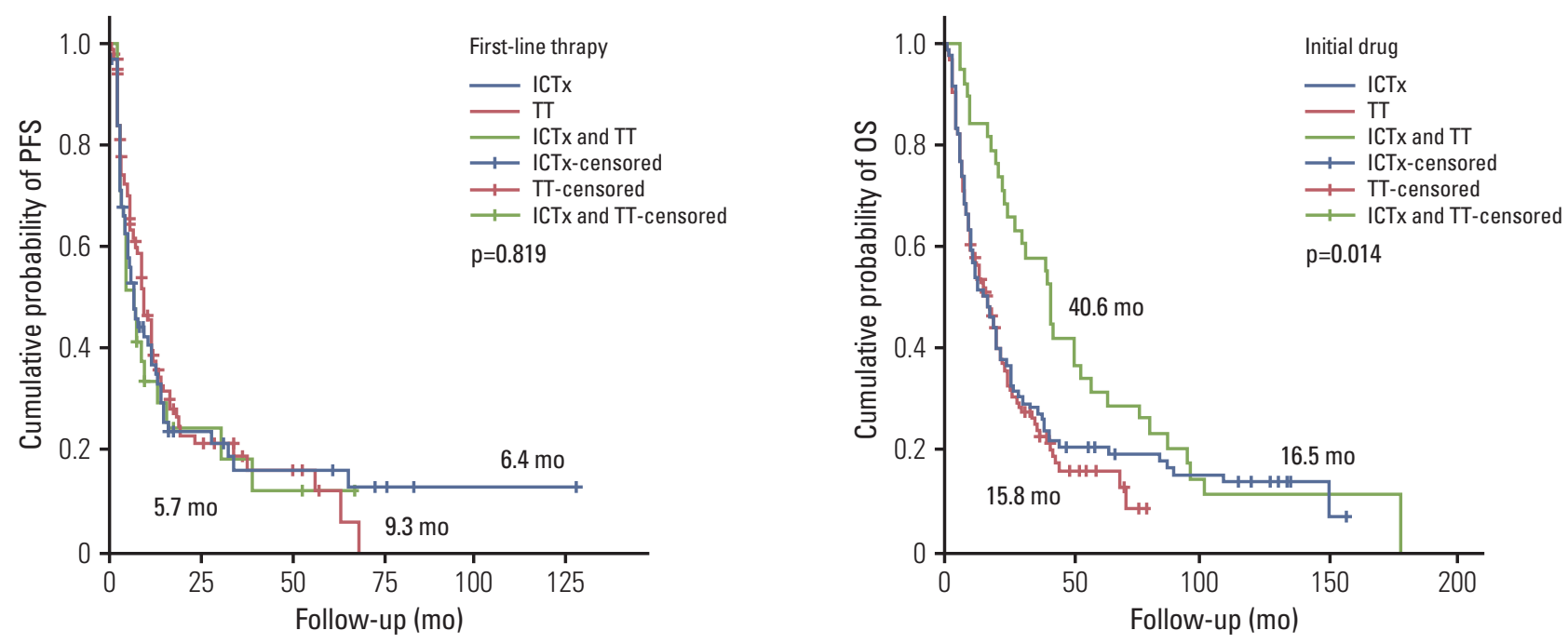

Fig. 1. Progression-free survival (PFS) (A) and overall survival (OS) (B) curves for first-line systemic therapies in patients with metastatic renal cell carcinoma treated with immunotherapy alone, targeted therapy alone, or both therapies in sequence. ICTx, immunotherapy; TT, targeted therapy.

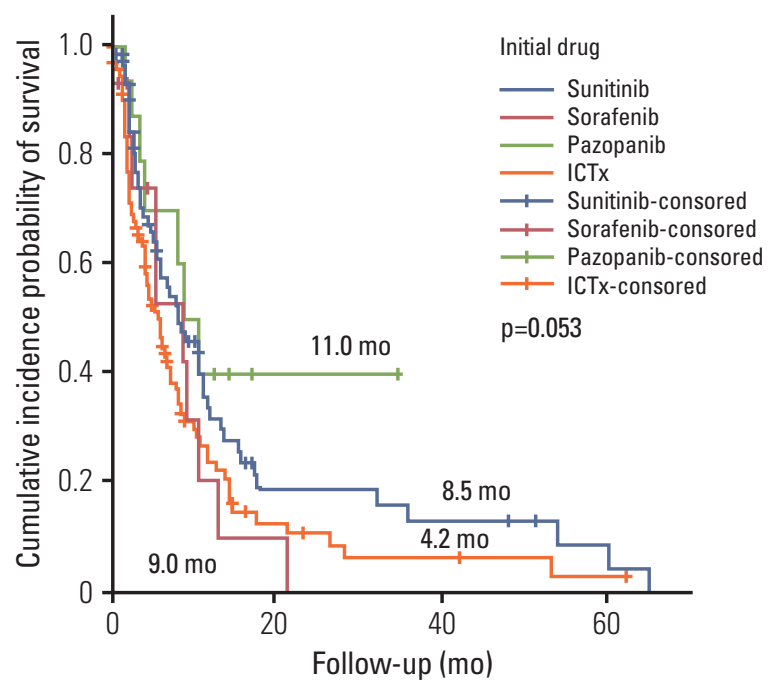

Fig. 2. Progression-free survival curves for first-line systemic therapies in patients with metastatic renal cell carcinoma. ICTx, immunotherapy.

when evaluating the prognosis of mRCC patients treated with sub-sequential TT.

In recent decades, compared with IT, multiple single TTs have shown greater activity in terms of PFS, specifically when compared with IFN- $\alpha[7,13]$. However, a clinically relevant increase in CR and OS has not been reported and the role of TT in increasing the curability of $\mathrm{mRCC}$ has not been fully elucidated. From a pharmacological viewpoint, the unsatisfactory results of TT to induce a CR may be explained in part by their mechanism of action, which appears to be more cytostatic than cytotoxic [15]. The current study also showed that the OS (15.8 months), ORR (33.9\%), and CR $(5.6 \%)$ of TT were either similar or inferior to the OS (16.5 months), ORR (31.1\%), and CR (10.4\%) of IT (Table 2).

The percentage of patients reaching CR for sunitinib, sorafenib, and pazopanib as first-line TT was reported as $1 \%-3 \%$ in earlier real-world clinical trials, post-marketing allpatient surveillance trials, and pivotal trials [16-18]. The higher CR rate $(5.6 \%)$ in this study would actually be much lower than observed because patients who stopped therapy after achieving CR during a substantial follow-up had a higher rate of relapse than patients who continued sequential therapy, as shown in a recent, large, retrospective study evaluating $\mathrm{mRCC}$ patients who were in CR during tyrosine kinase inhibitor treatment (either alone or in combination with local treatment) [19]. Another possible explanation for achievement of high $\mathrm{CR}$ would be that it is often integrated with TT with surgery, radiotherapy, or both. Johannsen et al. [20] reported a similarly increased CR rate in comparison with the current study in patients treated with tyrosine kinase inhibitors plus surgery $(4.5 \%)$ when compared to 


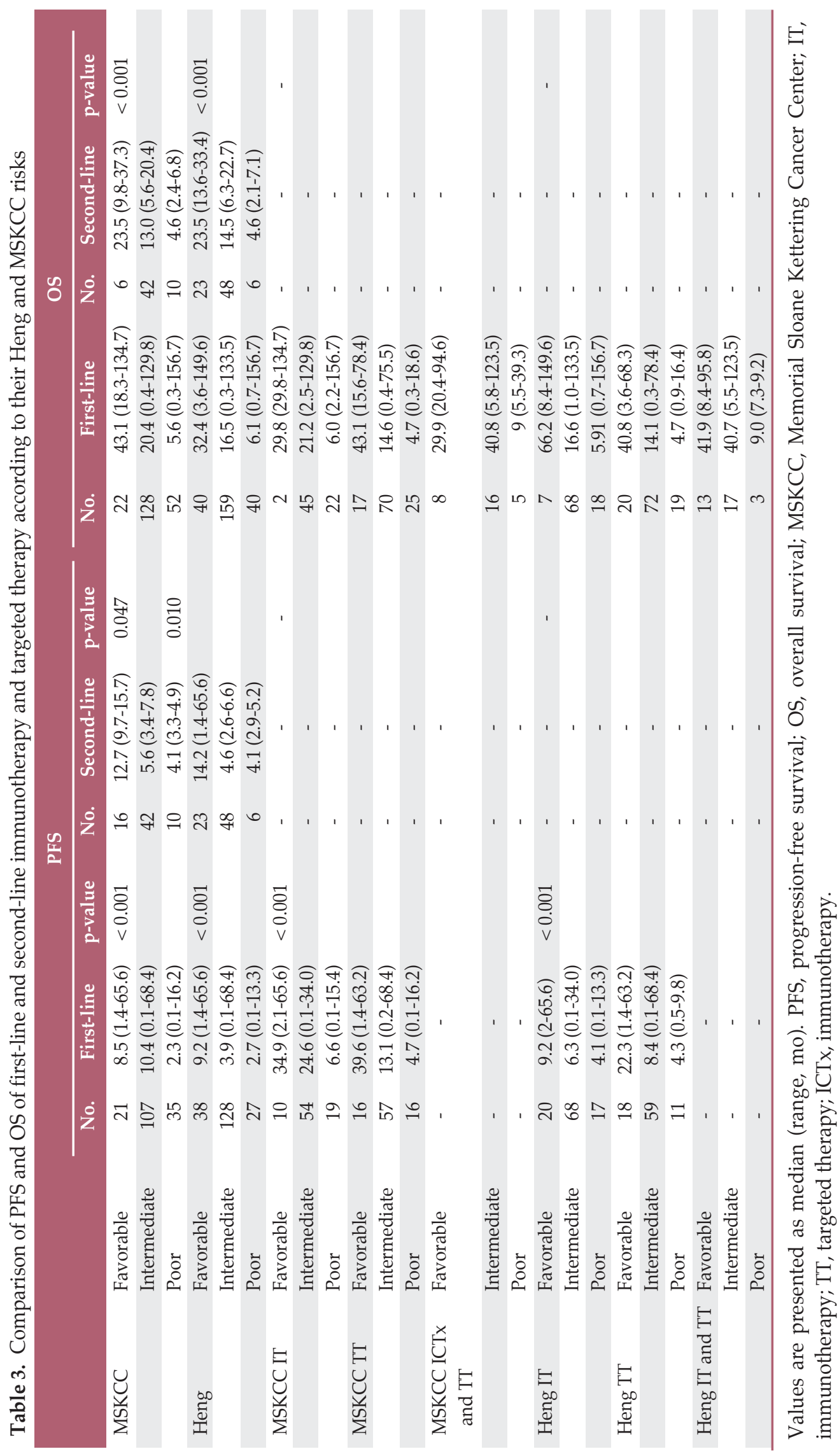


those treated with medical therapy alone (1.8\%). The current study also showed a significantly longer PFS (11.2 months) and greater CR rates (5.4\%) in patients who underwent nephrectomy plus TT compared to patients who underwent TT alone (PFS, 5.6 months; CR rate, 1.0\%; data not shown in tables).

Previous extended-access and post-marketing all-patient surveillance trials have reported $16-22 \% / 25.9 \% / 39 \%$ of ORRs and 5.2-9.4/6.0/13.7 months of PFSs for sunitinib, sorafenib, and pazopanib in mRCC patients, respectively [16-18,21]. The ORRs for sunitinib, sorafenib, and pazopanib reported in the current study were superior to those reported in previous studies $(44.1 \%, 27.3 \%$, and $62.5 \%$, respectively; data not shown); except for the PFS inferior or similar to previous studies (8.5, 9.0, and 11.0 months, respectively) [16-18,21].

Additional evaluation of second-line systemic therapies for their ORR, PFS, and OS (27.5\%, 6.5 months, and 15.3 months, respectively) was similar or superior to that of previous trials with second-line therapy in cytokine- or TT-refractory mRCC patients (ORR, 9\% to 23\%; PFS, 4 to 7.4 months; OS, 11.5 to 16.3 months) [7]. These differences of ORR, PFS, and OS are likely due to follow-up time, differences in clinical policies between clinicians surrounding various treatment modalities, differences in baseline study populations such as disease burden, nephrectomy rate, and physical body composition, as has been previously demonstrated for other malignancies $[22,23]$.

Regarding the analyses of prognostic models, patients with favorable MSKCC or Heng risk group showed longer survival [24]. In agreement, in the current study, those with favorable MSKCC or Heng risk group of first- and secondline therapy had a significantly better prognosis compared to those with poor or intermediate risk group (Table 3). When stratified according to MSKCC and Heng criteria, the PFS for all groups in the first-line therapy was better in those treated with TT compared to those treated with IT. However, those with a favorable Heng risk group treated with IT showed a superior OS in the first-line therapy, compared to all other groups and treatments.

There are some limitations of the current study. It was a retrospective study with a small patient population, with a possible patient selection bias based on the era of when systemic therapy was initiated. The relevance of the stratified findings based on MSKCC and Heng criteria is not fully understood and should be developed further in future largescale multicenter studies with a more diverse patient population.

\section{Conclusion}

Although the ORR of TT and IT were comparable in this study, TT demonstrated superior PFS and OS compared with IT in patients with mRCC. This supports the hypothesis that the clinical outcomes of patients with $\mathrm{mRCC}$ have shown significant improvement since the introduction of TT.

\section{Conflicts of Interest}

Conflict of interest relevant to this article was not reported.

\section{Acknowledgments}

This work was supported by a grant from the National Cancer Center (1510650-1), Republic of Korea.

\section{References}

1. Motzer RJ, Agarwal N, Beard C, Bolger GB, Boston B, Carducci MA, et al. NCCN clinical practice guidelines in oncology: kidney cancer. J Natl Compr Canc Netw. 2009;7:618-30.

2. Oudard S, George D, Medioni J, Motzer R. Treatment options in renal cell carcinoma: past, present and future. Ann Oncol. 2007;18 Suppl 10:x25-31.

3. Fossa SD, Raabe N, Moe B. Recombinant interferon-alpha with or without vinblastine in metastatic renal carcinoma: results of a randomised phase II study. Br J Urol. 1989;64:468-71.

4. Pyrhonen S, Salminen E, Ruutu M, Lehtonen T, Nurmi M,
Tammela T, et al. Prospective randomized trial of interferon alfa-2a plus vinblastine versus vinblastine alone in patients with advanced renal cell cancer. J Clin Oncol. 1999;17:2859-67.

5. Fisher RI, Rosenberg SA, Fyfe G. Long-term survival update for high-dose recombinant interleukin-2 in patients with renal cell carcinoma. Cancer J Sci Am. 2000;6 Suppl 1:S55-7.

6. Fyfe G, Fisher RI, Rosenberg SA, Sznol M, Parkinson DR, Louie AC. Results of treatment of 255 patients with metastatic renal cell carcinoma who received high-dose recombinant interleukin-2 therapy. J Clin Oncol. 1995;13:688-96. 
7. Cho IC, Chung J. Current status of targeted therapy for advanced renal cell carcinoma. Korean J Urol. 2012;53:217-28.

8. Lee JH, Chang SG, Jeon SH, Min GE, Yoo KH. Comparative analysis between immunochemotherapy and target therapy for metastatic renal cell carcinoma: overview of treatmentrelated adverse events and the dropout rate in Korea. Korean J Urol. 2010;51:379-85.

9. Heng DY, Xie W, Regan MM, Warren MA, Golshayan AR, Sahi C, et al. Prognostic factors for overall survival in patients with metastatic renal cell carcinoma treated with vascular endothelial growth factor-targeted agents: results from a large, multicenter study. J Clin Oncol. 2009;27:5794-9.

10. Motzer RJ, Bacik J, Murphy BA, Russo P, Mazumdar M. Interferon-alfa as a comparative treatment for clinical trials of new therapies against advanced renal cell carcinoma. J Clin Oncol. 2002;20:289-96.

11. Motzer RJ, Russo P. Systemic therapy for renal cell carcinoma. J Urol. 2000;163:408-17.

12. McDermott DF. Update on the application of interleukin-2 in the treatment of renal cell carcinoma. Clin Cancer Res. 2007;13(2 Pt 2):716s-20s.

13. Escudier B, Szczylik C, Hutson TE, Demkow T, Staehler M, Rolland F, et al. Randomized phase II trial of first-line treatment with sorafenib versus interferon Alfa-2a in patients with metastatic renal cell carcinoma. J Clin Oncol. 2009;27:1280-9.

14. Hutson TE, Davis ID, Machiels JP, De Souza PL, Rottey S, Hong BF, et al. Efficacy and safety of pazopanib in patients with metastatic renal cell carcinoma. J Clin Oncol. 2010;28: 475-80.

15. Desai AA, Stadler WM. Novel kinase inhibitors in renal cell carcinoma: progressive development of static agents. Curr Urol Rep. 2006;7:16-22.

16. Akaza H, Naito S, Ueno N, Aoki K, Houzawa H, Pitman Lowenthal S, et al. Real-world use of sunitinib in Japanese patients with advanced renal cell carcinoma: efficacy, safety and biomarker analyses in 1689 consecutive patients. Jpn J Clin Oncol. 2015;45:576-83.

17. Akaza H, Oya M, Iijima M, Hyodo I, Gemma A, Itoh H, et al.
A large-scale prospective registration study of the safety and efficacy of sorafenib tosylate in unresectable or metastatic renal cell carcinoma in Japan: results of over 3200 consecutive cases in post-marketing all-patient surveillance. Jpn J Clin Oncol. 2015;45:953-62.

18. Gore ME, Szczylik C, Porta C, Bracarda S, Bjarnason GA, Oudard $S$, et al. Final results from the large sunitinib global expanded-access trial in metastatic renal cell carcinoma. Br J Cancer. 2015;113:12-9.

19. Albiges L, Oudard S, Negrier S, Caty A, Gravis G, Joly F, et al. Complete remission with tyrosine kinase inhibitors in renal cell carcinoma. J Clin Oncol. 2012;30:482-7.

20. Johannsen M, Staehler M, Ohlmann CH, Florcken A, Schmittel $\mathrm{A}$, Otto $\mathrm{T}$, et al. Outcome of treatment discontinuation in patients with metastatic renal cell carcinoma and no evidence of disease following targeted therapy with or without metastasectomy. Ann Oncol. 2011;22:657-63.

21. Matrana MR, Bathala T, Campbell MT, Duran C, Shetty A, Teegavarapu $\mathrm{P}$, et al. Outcomes of unselected patients with metastatic clear-cell renal cell carcinoma treated with frontline pazopanib therapy followed by vascular endothelial growth factor receptor tyrosine kinase inhibitors (VEGFR-TKI) or mammalian target of rapamycin inhibitors (mTORi): a single institution experience. BJU Int. 2015 Nov 17 [Epub]. http://dx.doi.org/10.1111/bju.13374.

22. Cheng AL, Kang YK, Chen Z, Tsao CJ, Qin S, Kim JS, et al. Efficacy and safety of sorafenib in patients in the Asia-Pacific region with advanced hepatocellular carcinoma: a phase III randomised, double-blind, placebo-controlled trial. Lancet Oncol. 2009;10:25-34.

23. Ward E, Jemal A, Cokkinides V, Singh GK, Cardinez C, Ghafoor A, et al. Cancer disparities by race/ethnicity and socioeconomic status. CA Cancer J Clin. 2004;54:78-93.

24. Sadeghi S, Albiges L, Wood LS, Black SL, Gilligan TD, Dreicer $\mathrm{R}$, et al. Cessation of vascular endothelial growth factor-targeted therapy in patients with metastatic renal cell carcinoma: feasibility and clinical outcome. Cancer. 2012;118:3277-82. 